\title{
Adrenal suppression in patients taking inhaled glucocorticoids is highly prevalent and management can be guided by morning cortisol
}

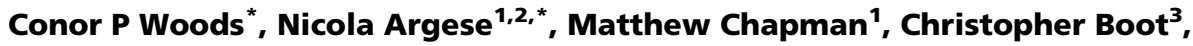 \\ Rachel Webster ${ }^{3}$, Vijay Dabhi ${ }^{4}$, Ashley B Grossman, Andrew A Toogood', \\ Wiebke Arlt' ${ }^{1}$, Paul M Stewart ${ }^{5}$, Rachel K Crowley ${ }^{1}$ and Jeremy W Tomlinson
}

Oxford Centre for Diabetes Endocrinology and Metabolism (OCDEM), NIHR Biomedical Research Centre, Churchill Hospital, University of Oxford, Headington, Oxford OX3 7L, UK, ${ }^{1}$ Centre for Endocrinology, Diabetes and Metabolism, University of Birmingham, University Hospitals Birmingham NHS Foundation Trust, Birmingham B15 2TH, UK, ${ }^{2}$ Department of Endocrinology, Faculty of Medicine and Psychology, St Andrea Hospital, Sapienza University of Rome, Rome, Italy, Departments of ${ }^{3}$ Biochemistry and ${ }^{4}$ Health Informatics, Queen Elizabeth Hospital, University Hospitals Birmingham NHS Foundation Trust, Birmingham B15 2TH, UK and ${ }^{5}$ Department of Endocrinology, University of Leeds, Leeds, UK

${ }^{*} \mathrm{C}$ P Woods and N Argese are joint first authors

\author{
Correspondence \\ should be addressed \\ to J W Tomlinson \\ Email \\ jeremy.tomlinson@ \\ ocdem.ox.ac.uk
}

\begin{abstract}
Context: Up to $3 \%$ of US and UK populations are prescribed glucocorticoids (GC). Suppression of the hypothalamo-pituitaryadrenal axis with the potential risk of adrenal crisis is a recognized complication of therapy. The $250 \mu \mathrm{g}$ short Synacthen stimulation test (SST) is the most commonly used dynamic assessment to diagnose adrenal insufficiency. There are challenges to the use of the SST in routine clinical practice, including both the staff and time constraints and a significant recent increase in Synacthen cost.

Methods: We performed a retrospective analysis to determine the prevalence of adrenal suppression due to prescribed GCs and the utility of a morning serum cortisol for rapid assessment of adrenal reserve in the routine clinical setting.

Results: In total, 2773 patients underwent 3603 SSTs in a large secondary/tertiary centre between 2008 and 2013 and $17.9 \%$ ( $n=496$ ) failed the SST. Of 404 patients taking oral, topical, intranasal or inhaled GC therapy for non-endocrine conditions, $33.2 \%(n=134)$ had a subnormal SST response. In patients taking inhaled GCs without additional GC therapy, 20.5\% (34/166) failed an SST and suppression of adrenal function increased in a dose-dependent fashion. Using receiver operating characteristic curve analysis in patients currently taking inhaled GCs, a basal cortisol $\geq 348 \mathrm{nmol} / \mathrm{l}$ provided $100 \%$ specificity for passing the SST; a cortisol value $<34 \mathrm{nmol} / \mathrm{l}$ had $100 \%$ sensitivity for SST failure. Using these cut-offs, $50 \%(n=83)$ of SSTs performed on patients prescribed inhaled GCs were unnecessary.
\end{abstract}

Conclusion: Adrenal suppression due to GC treatment, particularly inhaled GCs, is common. A basal serum cortisol concentration has utility in helping determine which patients should undergo dynamic assessment of adrenal function.

\section{Introduction}

Glucocorticoids (GC) have a diverse array of functions affecting every tissue in the body. GCs are essential for life, and both excess and deficiency are associated with increased mortality $(1,2)$. In patients with an intact hypothalamo-pituitary-adrenal (HPA) axis, endogenous secretion of cortisol from the adrenal cortex is under the control of adrenocorticotropic hormone (ACTH) from the anterior pituitary $(3,4)$. The dynamic function of the HPA (c) 2015 The authors Published by Bioscientifica Ltd

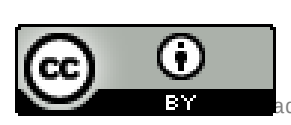

This work is licensed under a Creative Commons Attribution 3.0 Unported License. 
axis is most frequently assessed in clinical practice using the $250 \mu \mathrm{g}$ short Synacthen (corticotropin) stimulation test (SST). The SST has been validated against the gold standard of insulin-induced hypoglycaemia to provide an accurate reflection of adrenal cortisol reserve (5), therefore representing the most widely used tool for diagnosing adrenal insufficiency. There are significant challenges to the use of the SST in clinical practice. These challenges include both the staff and time needed to perform the SST, a well-documented recent (2014) shortage of Synacthen and a subsequent significant increase in Synacthen cost.

Endocrine diseases affecting the adrenal or pituitary gland are important causes of primary and secondary causes of adrenal insufficiency respectively (6). However, prescribed GCs can also cause long-term suppression of the HPA axis and, consequently, adrenal atrophy and an inability to mount an adequate cortisol response to stress, rendering patients at an increased risk of adrenal crisis. Adrenal crisis is a medical emergency necessitating hospital admission, resuscitation with i.v. fluids and parenteral GC treatment, and is associated with increased mortality and a poor outcome $(7,8)$.

The therapeutic indications and clinical benefit associated with GC treatment prescribed for underlying inflammatory conditions are not in doubt. Estimates suggest that up to $3 \%$ of the population of the UK and USA are currently taking GC therapy $(9,10)$. Long-term prescription rates for oral GCs have increased, as has the use of inhaled GC therapy (11). In the majority of cases, the doses of prescribed GCs are sufficient to cause HPA axis suppression (total daily doses $>5 \mathrm{mg}$ prednisolone or equivalent) (10). GC prescriptions are often extended for a sustained period of time with a median duration in excess of 4 years; in $25 \%$ of the cases, treatment extends beyond 5 years (9). As a result, suppression of the HPA axis may be frequent and potentially overlooked. Dynamic assessment of the HPA axis using the SST has not been widely reported in the literature in the setting of iatrogenic adrenal suppression. Furthermore, the decision to undertake dynamic HPA axis testing in patients taking prescribed GCs is often based upon the presence of symptoms that may be suggestive of adrenal insufficiency, but these can be vague and often non-specific (12). Importantly, there are currently no published data to help guide the clinician to decide in which patients to undertake assessment of adrenal cortisol reserve. We can assume that people taking a regular dose of $5 \mathrm{mg}$ of prednisolone (or equivalent) have compromised adrenal reserve; however, the degree of HPA axis suppression in persons taking inhaled, topical or intra-articular GCs is unknown.
We have performed a detailed retrospective analysis over a 5-year period in a secondary/tertiary care centre. Firstly, we have aimed to determine the prevalence of adrenal insufficiency in patients undergoing SSTs across all medical specialties, and specifically the prevalence of adrenal insufficiency due to prescribed GCs. Secondly, we have analysed whether a morning serum cortisol can guide the clinician as to which patients are likely to have an intact HPA axis, and thus to rationalize further investigation and management.

\section{Methods}

We retrospectively analysed the results of SSTs performed in 2773 patients from the electronic medical record system at the Queen Elizabeth Hospital, University Hospitals Birmingham NHS Foundation Trust, UK. All SSTs were performed in the morning (0900-1200 h), between $1 / 1 / 2008$ and 31/12/2012 across all specialties in a single large secondary/tertiary care centre in the UK. An i.m. administration of synthetic ACTH [1-24] was used (Synacthen $250 \mu \mathrm{g}$, Alliance Pharmaceuticals, Chippenham, UK). In patients treated with oral GCs, these were suspended for 24 -h prior to testing. For patients with acute pituitary insult (surgery or apoplexy), the SST was delayed for a minimum of at least 4 weeks after surgery or presentation, as earlier assessment can result in false positive passes, since adrenal atrophy only develops 4 weeks after the insult (13). Clinical characteristics of individuals were recorded, including age, sex, indication for the SST and the use of GC medication (including dose and route of administration). Patients on GC replacement therapy as a consequence of adrenal or pituitary disease were classified according to their underlying endocrine condition rather than in groups associated with GC administration. Similarly, patients with CNS disease (including tumours) were classified separately from those prescribed GC therapy due to the potential for the underlying disease process to impact upon endogenous HPA axis function. Serum cortisol was analysed on a standard automated competitive immunoassay platform (Roche Modular system, Roche) where there is no evidence for cross-reactivity with commonly prescribed inhaled GCs (14). The inter-assay imprecision was $<8 \%$ for levels between 76 and $925 \mathrm{nmol} / \mathrm{l}$. The basal and stimulated 30-min cortisol samples were analysed in a single batch. Using data from a healthy control population (15) and the same cortisol assay, a 30-min cortisol of $\geq 550 \mathrm{nmol} / \mathrm{l}$ was regarded as demonstrating a satisfactory adrenal reserve 
and designated a 'pass', whereas a 30-min value of $<550 \mathrm{nmol} / \mathrm{l}$ was designated a 'fail'.

\section{Statistical analysis}

Results are expressed as means \pm s.D. for parametric data and medians and inter-quartile ranges for non-parametric data. For comparison of single variables, $t$-tests have been used (or non-parametric equivalents) with paired analysis where appropriate. For analyses involving multiple comparisons, one-way ANOVA with Bonferroni's multiple comparison post-hoc correction or, for non-parametric data, Kruskal-Wallis with Dunn's multiple correction tests were used to determine statistical significance. Two-tailed significance was set at $P<0.05$. For comparison of the prevalence of SST failure/pass, $\chi^{2}$ analysis was used. To analyse basal cortisol as a predictor of passing the SST, receiver operator characteristic (ROC) curves were generated with true positive results (sensitivity) plotted against false positive (1-specificity). Area under the curve (AUC) of a ROC curve indicates the ability to discriminate a true result, with values of 0.5 showing no discrimination and values of 1.0 equal to perfect discrimination. The 'best fit' value of the curve was determined using the Youden index (threshold value for which (Sensitivity + Specificity -100) is maximized). We performed sub-group analysis in the following categories: age, sex (including pre and postmenopause), pituitary disease and inhaled GCs. To determine if the inclusion of repeat tests would influence the discriminatory value of a morning cortisol to predict adrenal reserve, a further analysis including the entire cohort of all 3603 SSTs was performed (see Supplementary Table 1, see section on supplementary data given at the end of this article). Statistical analysis was performed using the GraphPad Prism 6.0 software package (GraphPad Software, Inc. La Jolla, CA, USA).

\section{Results}

Overall, 496 patients (17.9\%) failed the SST (Table 1). In cases where the SST had been performed without a confirmed endocrine diagnosis, or in patients not receiving GC therapy, failure rates were low (0-8.8\%).

\section{Adrenal suppression due to prescribed GCs}

In total, 404 SSTs were performed to determine adrenal reserve in patients who had been prescribed GC therapy (indications 1 and 2 of Table 1). The prevalence of SST failure in this cohort was 33.2\% (134/404). Patients with pituitary, adrenal and CNS disease (indications 3-8 from Table 1) were then excluded. Patients were further subdivided based upon their GC therapy status: those currently taking GCs and those previously exposed to GC therapy (Table 2). Data were compared against individuals who were GC naïve and without established endocrine or CNS disease $(n=1287)$. In GC-naïve patients, $7.5 \%$ (96/1287) failed the SST. A similar SST failure rate was observed for those patients previously exposed to GC therapy $(10.9 \%(7 / 64))$. However, failure rates were significantly higher in patients currently taking GC therapy $\left(37.4 \%(127 / 340) \chi^{2}\right.$ analysis, $\left.P<0.05\right)$. In patients

Table 1 The results of SSTs in 2773 patients divided according to indication.

\begin{tabular}{|c|c|c|c|c|}
\hline & Description of indication & Total $(n)$ & $\%$ Pass $(n)$ & $\%$ Fail $(n)$ \\
\hline 1 & Treatment with inhaled, intra-nasal or topical glucocorticoids & 228 & $75.4(172)$ & $24.6(56)$ \\
\hline 2 & Treatment with oral or i.v. glucocorticoids & 176 & $55.7(98)$ & $44.3(78)$ \\
\hline 3 & $\begin{array}{l}\text { Post-operative assessment after pituitary surgery (without } \\
\text { radiotherapy) }\end{array}$ & 195 & 70.3 (137) & 29.7 (58) \\
\hline 4 & Post-operative assessment after pituitary surgery (with radiotherapy) & 59 & $71.2(42)$ & $28.8(17)$ \\
\hline 5 & Pituitary adenoma (without surgery or radiotherapy) & 264 & $89.8(237)$ & $10.2(27)$ \\
\hline 6 & Other conditions affecting the pituitary & 175 & $77.1(135)$ & $22.9(40)$ \\
\hline 7 & Other tumours of the CNS & 315 & $74.9(236)$ & $25.1(79)$ \\
\hline 8 & Adrenal disease (CAH, Addison's disease, adenoma, carcinoma) & 74 & $39.2(29)$ & $60.8(45)$ \\
\hline 9 & $\begin{array}{l}\text { Co-existent autoimmune disease (thyroid disease, type } 1 \text { diabetes } \\
\text { mellitus, premature ovarian failure, vitiligo) }\end{array}$ & 113 & $92.0(104)$ & $8.0(9)$ \\
\hline 10 & Hyponatraemia or hyperkalaemia & 68 & $91.2(62)$ & $8.8(6)$ \\
\hline 11 & Hypoglycaemia & 32 & $100(32)$ & $0(0)$ \\
\hline 12 & Hypotension, syncope, collapse & 173 & $96.0(166)$ & $4.0(7)$ \\
\hline 13 & Fatigue, weight loss, malaise & 178 & $92.7(165)$ & $7.3(13)$ \\
\hline 14 & Other indications, including critical care admission or not specified & $\begin{array}{r}723 \\
2773\end{array}$ & $\begin{array}{c}91.6(662) \\
82.1 \%(2277)\end{array}$ & $\begin{array}{c}8.4(61) \\
17.9 \%(496)\end{array}$ \\
\hline
\end{tabular}


Table 2 The impact of glucocorticoid therapy status upon SST results. Indications are as follows: (1) treatment with inhaled, nasal or topical glucocorticoids; (2) treatment with i.v. or oral glucocorticoids; (3) post-operative assessment after pituitary surgery (without radiotherapy); (4) post-operative assessment after pituitary surgery (with radiotherapy); (5) pituitary adenoma (without surgery or radiotherapy); (6) other conditions affecting the pituitary; (7) other tumours of the CNS; (8) Adrenal disease (CAH, Addison's disease, adenoma, carcinoma); (9) co-existent autoimmune disease (thyroid disease, type 1 diabetes mellitus, premature ovarian failure, vitiligo); (10) hyponatremia or hyperkalaemia; (11) hypoglycaemia; (12) hypotension, syncope, collapse; (13) fatigue, weight loss, malaise; and (14) other indications, including critical care admission or not specified.

\begin{tabular}{l}
\hline \\
Indication \\
\hline 1 \\
2 \\
3 \\
4 \\
5 \\
6 \\
7 \\
8 \\
9 \\
10 \\
11 \\
12 \\
13 \\
14
\end{tabular}

\begin{tabular}{rr} 
& Gluco \\
\hline$n$ \\
\hline 0 \\
0 \\
103 \\
44 \\
238 \\
132 \\
222 \\
34 \\
113 \\
68 \\
32 \\
173 \\
178 \\
723
\end{tabular}

lucocorticoid naive

$\%$ Pass $(n)$

$\%$ Fail $(n)$

0
0

$89.3(92)$

0
0
$10.7(11)$
$13.6(6)$
$5.0(12)$
$6.8(9)$
$5.0(11)$
$23.5(8)$
$8.0(9)$
$8.8(6)$
$0(0)$
$4.0(7)$
$7.3(13)$
$8.4(61)$

\begin{tabular}{r} 
Previous \\
\hline$n$ \\
\hline 13 \\
51 \\
9 \\
2 \\
3 \\
1 \\
18 \\
0 \\
0 \\
0 \\
0 \\
0 \\
0 \\
0
\end{tabular}

currently taking prescribed GCs, failure rates were highest in those patients taking oral therapy (58.4\% (73/125), $P<0.01)$.

\section{HPA axis suppression and inhaled GCs}

SST failure remained high in patients currently prescribed inhaled GC or topical GC therapy compared to GC-naïve patients (Fig. 1). In patients taking inhaled GCs without additional GC therapy, 34 of 166 patients failed the SST (20.5\%). SST failure rates were $21.2 \%(22 / 104)$ for patients taking fluticasone, $16.7 \%$ (6/36) for beclometasone, $19.1 \%$ $(4 / 21)$ for budesonide and $2 / 5$ on ciclesonide. Where doses were recorded (beclometasone $=28$, or fluticasone $n=79$ ), the prevalence of SST failure was highest in those patients taking the largest doses (Fig. 1C and D). The 30-min serum cortisol levels were significantly lower in patients on the highest doses of both beclometasone and fluticasone in comparison with those patients on lower doses (Fig. 1E and F). Baseline cortisol levels were also suppressed on the highest doses of fluticasone $(461 \pm 60$ vs $205 \pm 49 \mathrm{nmol} / \mathrm{l}$, $P<0.05$ ) (Fig. 1F).

Comparing the SST response between patients taking beclometasone or fluticasone, 30-min cortisol levels were not significantly different at the highest doses of inhaled GCs $(445 \pm 85$ vs $631 \pm 98 \mathrm{nmol} / \mathrm{l}$, fluticasone $>500 \mu \mathrm{g} /$ day vs beclometasone $>400 \mu \mathrm{g} /$ day, $P=\mathrm{NS}$ ).

\begin{tabular}{|c|c|c|}
\hline \multicolumn{3}{|c|}{ Current glucocorticoid therapy } \\
\hline$n$ & $\%$ Pass $(n)$ & $\%$ Fail $(n)$ \\
\hline 215 & $74.9(161)$ & $25.1(54)$ \\
\hline 125 & $41.6(52)$ & $58.4(73)$ \\
\hline 83 & $43.4(36)$ & $56.6(47)$ \\
\hline 13 & $15.4(2)$ & $84.6(11)$ \\
\hline 23 & $43.5(10)$ & $56.5(13)$ \\
\hline 42 & $26.2(11)$ & $73.8(31)$ \\
\hline 75 & $13.3(10)$ & $86.7(65)$ \\
\hline 40 & $7.5(3)$ & $92.5(37)$ \\
\hline 0 & 0 & 0 \\
\hline 0 & 0 & 0 \\
\hline 0 & 0 & 0 \\
\hline 0 & 0 & 0 \\
\hline 0 & 0 & 0 \\
\hline 0 & 0 & 0 \\
\hline
\end{tabular}

However, at lower doses, fluticasone therapy was associated with a greater impairment of 30-min cortisol response $(858 \pm 70$ vs $1229 \pm 201 \mathrm{nmol} / \mathrm{l}$, fluticasone $<500 \mu \mathrm{g} / \mathrm{day}$ vs beclometasone $<400 \mu \mathrm{g} /$ day, $P<0.05 ; 740 \pm 37$ vs $1009 \pm 177 \mathrm{nmol} / \mathrm{l}$, fluticasone $500 \mu \mathrm{g} /$ day vs beclometasone $400 \mu \mathrm{g} /$ day, $P<0.05)$. There were no significant differences in clinical characteristics in the patient cohorts when comparing across prescribed dose of inhaled GCs between different inhaled GCs or those who failed or passed the SST (data not shown).

\section{Baseline morning cortisol as a predictor of SST outcome}

Baseline serum cortisol in the cohort of 3606 SSTs (2773 patients) correlated significantly with the levels $30 \mathrm{~min}$ after ACTH stimulation (Sperman's $\rho=+0.74 P<0.0001$ ). ROC curve analysis of baseline morning cortisol as a predictor for passing the SST showed an AUC of 0.91 . Serum cortisol concentration of $506 \mathrm{nmol} / \mathrm{l}$ gave $100 \%$ specificity for predicting passing the SST and a serum cortisol concentration of $107 \mathrm{nmol} / \mathrm{l}$ was $99 \%$ sensitive for predicting failure (Table 3 ). In the 404 patients exposed to GC therapy, a serum cortisol concentration of $410 \mathrm{nmol} / \mathrm{l}$ gave $100 \%$ specificity for predicting passing the SST and a serum cortisol concentration of $34 \mathrm{nmol} / \mathrm{l}$ was $100 \%$ sensitive for predicting failure. Also, 166 SSTs were performed on patients taking inhaled GCs. All those 

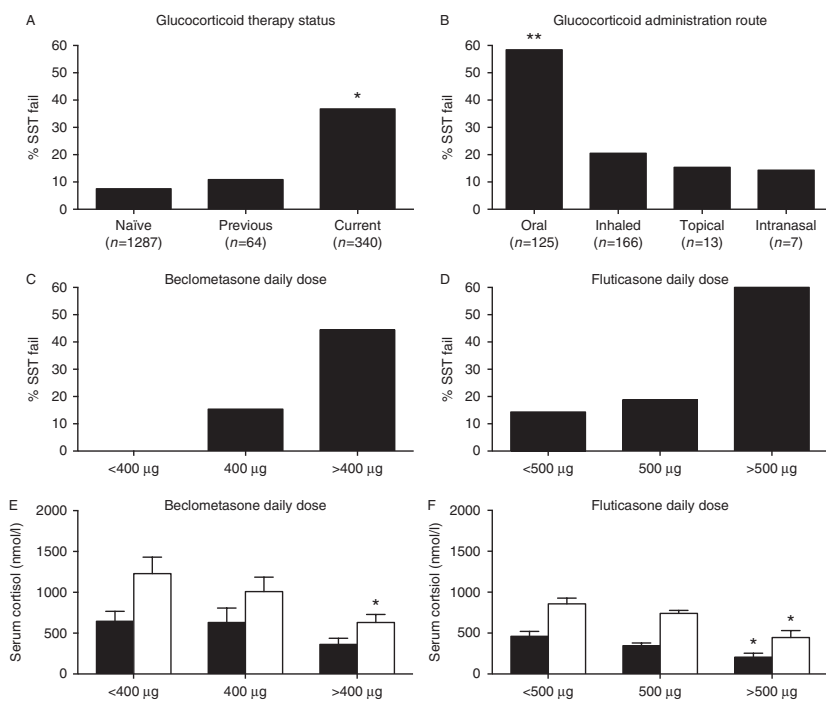

\section{Figure 1}

Excluding patients with underlying pituitary, adrenal or CNS disease, current glucocorticoid therapy is associated with increased rates of SST failure (A). SST failure is common across all routes of glucocorticoid administration, although it is most frequent in patients on oral therapy (B). ( $\left.{ }^{*} P<0.05, * * P<0.01\right)$. The impact of inhaled glucocorticoid therapy on the prevalence of adrenal suppression (C, D, E and F). Beclometasone and fluticasone administration cause a dose-dependent increase in SST failure rates ( $C$ and $D$ ) and absolute reductions in basal and 30-min cortisol levels after synthetic $\mathrm{ACTH}_{1-24}$ stimulation (E and F) (basal serum cortisol= black bars, 30-min cortisol = white bars) ( ${ }^{*} P<0.05$ vs lowest daily dose).

with a baseline morning cortisol of $348 \mathrm{nmol} / \mathrm{l}$ or higher passed the SST and all those with a baseline serum cortisol concentration of $34 \mathrm{nmol} / 1$ or lower failed the SST (Table 3 and Fig. 2A). Adopting these 100\% cut-offs (348 and 34 respectively, 83 SSTs (representing $50 \%$ of all SSTs undertaken in patients on inhaled GCs) need not have been performed, as the results of the SST could have been predicted form the morning cortisol result alone (Fig. 2A).
The AUC, best-fit cortisol value and cut-off cortisol values for respective specificities and sensitivities according to age, sex, menopausal status and other indications (including endocrine diagnoses) are presented in Supplementary Table 2 , see section on supplementary data given at the end of this article.

\section{Discussion}

In this study we have demonstrated a high prevalence of adrenal insufficiency associated with GC treatment in the largest cohort described to date, with an alarming prevalence in those patients taking GC therapy for nonendocrine disease. Indeed, SST failure rates were higher in those taking GC therapy than in those with underlying pituitary disease. GCs are currently prescribed to up to $3 \%$ of the population $(9,10,11)$ and there is little doubt that GC excess is associated with significant adverse effects and suppression of the HPA axis (16). Furthermore, epidemiological data from general practice records has demonstrated a link between GC prescriptions and the risk of cardiovascular events and heart failure (17).

Adrenal suppression is considered unusual where GCs have been prescribed for $<2$ weeks (18) but is more likely following prolonged administration (19). However, a recent prospective study in patients prescribed high-dose oral prednisone $(40 \mathrm{mg})$ identified evidence of adrenal suppression in $>60 \%$ of patients after 6 days of therapy (20). Some studies have suggested that adrenal suppression is not tightly linked to the duration and dose of oral GCs $(16,21)$; however, this is not the case in all studies (22) and this may well reflect inter-individual variability in GC action and metabolism, including the activity of CYP3A4, the major pathway for the inactivation of most prescribed GCs $(23,24,25,26)$.

Within our cohort, more than $20 \%$ of patients taking prescribed inhaled GCs failed the SST, which is consistent with some but not all of the published literature $(27,28,29)$. The variability in results may reflect the use of inappropriate

Table 3 SST results with AUC, best-fit serum cortisol (max (Sensitivity + Specificity - 100)) and sensitivities and specificities for serum cortisol concentrations (nmol/l).

\begin{tabular}{ll}
\hline Group & \multicolumn{1}{c}{ AUC } \\
\cline { 1 - 2 } All patients $(n=2773)$ & $0.91(0.89-0.92)$ \\
All current GC use $(n=404)$ & $0.92(0.89-0.94)$ \\
Inhaled GCs $(n=166)$ & $0.90(0.85-0.95)$ \\
Oral GCs $(n=143)$ & $0.89(0.84-0.94)$ \\
\hline
\end{tabular}

\begin{tabular}{c}
\hline Best-fit cortisol (nmol/l) \\
\hline $221(86 \%$ sens $+77 \%$ spec $)$ \\
$220(78 \%$ sens $+88 \%$ spec $)$ \\
$308(70 \%$ sens $+94 \%$ spec $)$ \\
$213(74 \%$ sens $+94 \%$ spec $)$ \\
\hline
\end{tabular}

\begin{tabular}{ccccc}
\hline \multicolumn{3}{c}{ Specificity $(\%)$} \\
\hline 95 & & 99 & & 100 \\
\cline { 1 - 1 } 347 & & 461 & & 506 \\
331 & & 386 & & 410 \\
342 & & 347 & & 348 \\
383 & & 405 & & 413 \\
\hline
\end{tabular}

\begin{tabular}{|c|c|c|}
\hline \multicolumn{3}{|c|}{ Sensitivity (\%) } \\
\hline 95 & 99 & 100 \\
\hline 164 & 107 & $<20$ \\
\hline 168 & 63 & 34 \\
\hline 146 & 62 & 34 \\
\hline 198 & 115 & 114 \\
\hline
\end{tabular}



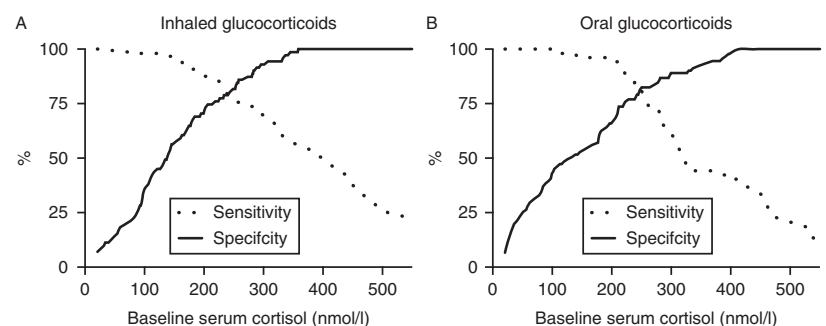

\section{Figure 2}

Baseline serum cortisol as a predictor of 30-min cortisol levels during an SST. Baseline serum cortisol is graphed against the \% likelihood of passing (specificity = continuous line) or failing (sensitivity=dashed line) the SST. (A) Inhaled glucocorticoids. (B) Oral glucocorticoids.

assessments of HPA axis and paucity of dynamic testing including SSTs; isolated measurements of serum cortisol are difficult to interpret and can be misleading. We and others have previously documented the prevalence of adrenal insufficiency in relatively small cohorts of patients $(n=33)$ taking inhaled GCs for respiratory disease and demonstrated SST failure rates of up to $48 \%$ (30).

Our data have shown a dose dependency in the SST response with inhaled GCs and are in agreement with evidence from the published literature suggesting that adrenal suppression is most frequent at the highest doses of inhaled GCs (31), although we did observe suppression in some patients taking lower doses. In addition, it is important to recognize that adrenal suppression can persist for prolonged periods after discontinuation of GC therapy (32). Whilst we have been able to demonstrate the magnitude of a clinical problem that is more extensive than is widely appreciated, it is fundamentally important to understand its clinical consequences. Current clinical data in this regard are lacking. We have previously shown that decreased adrenal reserve as a consequence of inhaled GCs is associated with reduced quality of life in patients with bronchiectasis (30) and the use of inhaled GCs in patients with chronic obstructive pulmonary disease (COPD) is known to increase the risk of type 2 diabetes and pneumonia $(11,33)$. In the setting of intensive care, a significant proportion of patients have functional adrenal insufficiency and this has been associated with a poorer outcome (34). Taken together, these observations raise the intriguing possibility that poor clinical outcome in patients on inhaled GCs may be due at least in part to HPA axis suppression, adrenal insufficiency and the consequent inability to mount an adequate cortisol stress response, and this has significant implications for the therapeutic approach to these patients (Supplementary Table 3 and Supplementary Figure 1, see section on supplementary data).

Routine screening for adrenal insufficiency in the context of inhaled GCs is not currently recommended. The magnitude of the clinical problem may be daunting, and making a case for routine assessment of HPA axis function in all patients on inhaled GCs may be prohibitive considering the workload and cost implications. To date, dynamic stimulation tests such as the SST and the ITT are considered mandatory for reliable investigation of adrenal reserve $(13,35,36)$. The utility of a basal cortisol in predicting adrenal reserve has been examined previously $(37,38)$. Using a single morning cortisol as the first screening step has the potential, in some circumstances, to reduce the need for dynamic tests and as a consequence to decrease cost and workload and reduce unnecessary tests for patients. In addition, the recent well-publicized worldwide shortage of Synacthen has highlighted the role that alternative assessments of HPA axis function may play. A baseline cortisol result below $100 \mathrm{nmol} / \mathrm{l}$ is considered highly indicative of adrenal insufficiency $(37,39)$, although this is not universally accepted $(35,40)$. Different basal cortisol levels, ranging from 285 to $500 \mathrm{nmol} / \mathrm{l}$, have been proposed as predictive of adequate adrenal reserve $(35,37,39,40,41,42,43)$. However, these studies have been small $(n=197,761,68,679,210,54$ and 166 respectively) and in many cases have not been able to systematically examine variable cortisol level cut-offs or determine the impact of underlying aetiology and specifically the clinical problem of iatrogenic adrenal insufficiency. Through our systematic approach to assessing adrenal function in a large cohort of patients, we are now able to propose an algorithm (Fig. 3) that is likely to have significant clinical utility and is safe. In patients prescribed inhaled GCs and in the absence of symptoms of adrenal insufficiency, we recommend measuring annual baseline serum cortisol concentrations as a useful tool in assessing adrenal GC reserve. A baseline serum cortisol $>348 \mathrm{nmol} / \mathrm{l}$ is suggestive of an intact adrenal response to stress. A baseline cortisol of $<35 \mathrm{nmol} / 1$ suggests the need for physiological oral hydrocortisone replacement and, if possible, a reduction in inhaled GC doses. Baseline serum cortisol concentrations between 35 and $348 \mathrm{nmol} / 1$ suggest the need for further investigation, including an SST and possible oral GC replacement. We deliberately identified a cut-off with $100 \%$ specificity such that we can be confident not to miss unrecognized adrenal insufficiency. Adopting this algorithm would mean that $50 \%$ of the SSTs on patients taking inhaled GCs need not have 
- Clinical suspicion of adrenal insufficiency

- Consider annual baseline cortisol for patients on high dose inhaled glucocorticoids

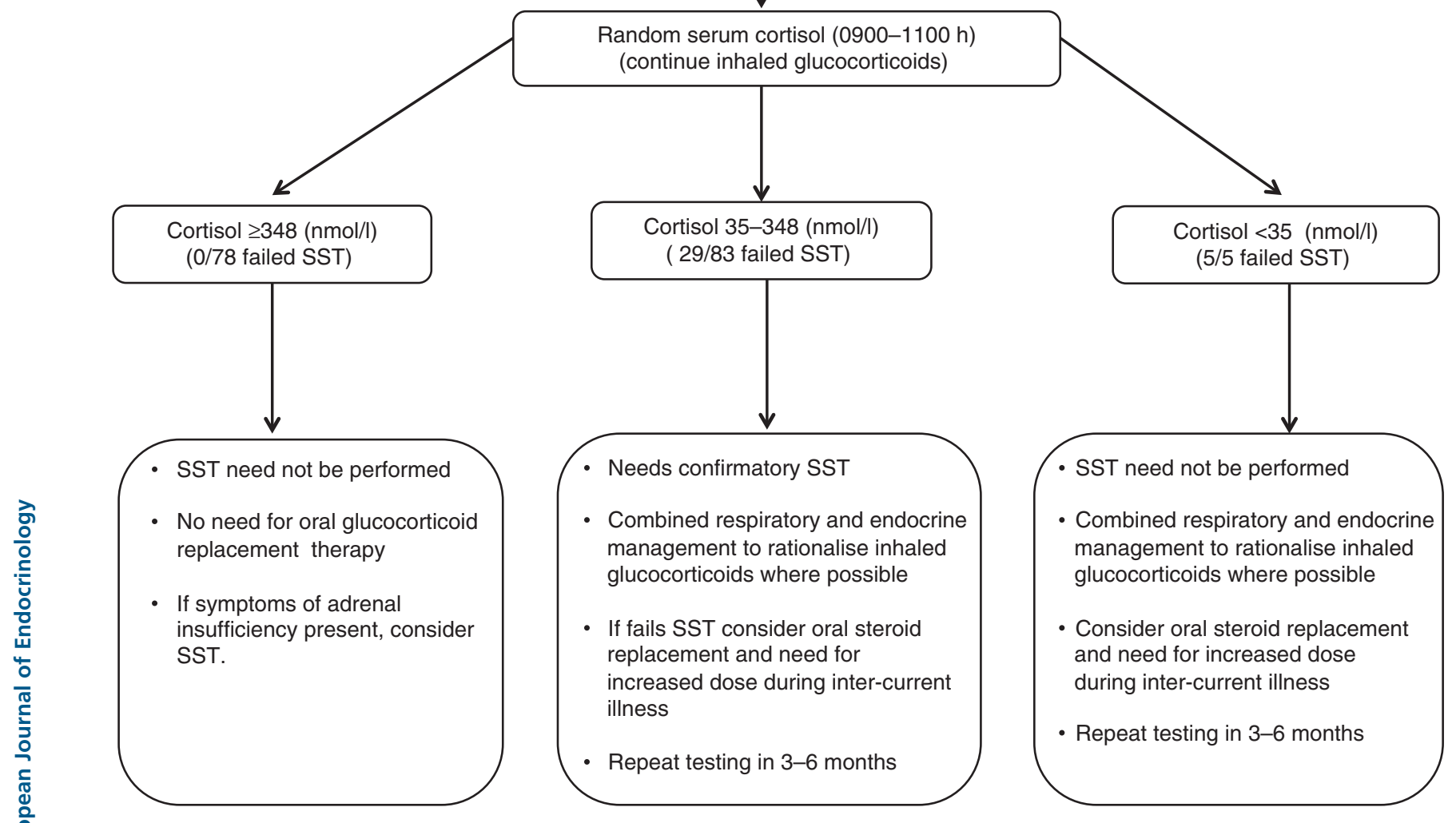

Figure 3

Putative algorithm to aid in the rationalization of assessment of the HPA axis in patients taking inhaled glucocorticoids.

been performed. A similar approach could be used in patients with underlying adrenal or pituitary pathology, limiting unnecessary test and the potential for significant cost savings.

The management of patients with adrenal insufficiency in the context of endocrine disease has been reviewed extensively (6). However, there are currently no guidelines or published studies that have determined the optimal management strategy for those patients with adrenal insufficiency due to prescribed GCs. For those on oral therapy, continuation of treatment as clinically indicated is appropriate, followed by weaning of the dose if the duration has been longer than 2 weeks. Once the daily doses have been reduced to those approaching physiological levels (equivalent of prednisolone $5 \mathrm{mg} /$ day), and assuming that GC therapy is no longer indicated for their initial prescribed purpose, an SST should be performed and, if passed, the GCs can be safely stopped. If, however, the SST is failed, we would normally initiate hydrocortisone replacement therapy with appropriate 'sick day rules' with regards to increasing dose at times of stress or inter-current illness, provide the patient with information and a 'steroid card' and perform repeat testing after 3-6 months.

For those patients taking inhaled GCs, the situation is more complex, as the inhaled GC cannot provide the adequate systemic actions that are needed at times of stress. A combined respiratory and endocrinology approach is appropriate. If possible, inhaled GC doses may be reduced, but this will be entirely dependent upon the underlying respiratory condition. For those patients who fail the SST, we would advocate daily hydrocortisone replacement (including advice with regards to 'sick day rules' and provision of an emergency 'steroid card' as 
described above). Repeat SST should be performed, especially if modification to the inhaled GC therapy has been undertaken to assess the potential return of HPA axis function.

There are limitations with the current analysis, although it does represent a cross-sectional analysis of unselected clinical data across all medical specialties. The data are retrospective and the decision to perform the SST was based on clinical indication as assessed by the individual clinician leading to a potential positive selection bias that may overestimate prevalence rates. Duration of GC treatment is likely to be important, as is the length of time since cessation of GC therapy. However, data from our electronic patient records does not allow accurate assessments of the duration of therapy or the time since therapy had been discontinued. The cut-off levels in this paper relate specifically to the assay used, and interpretation of these data should be made in the context of local cortisol assays as well as the methodology for the SST (i.m. vs i.v. Synacthen and 30 vs $60 \mathrm{~min}$ cortisol values). We advocate utilising the i.m. high dose Synacthen test, as it performs well in clinical practise $(13,44)$ and has been validated against the gold standard (insulin-induced hypoglycaemia) (5). In addition, robust longitudinal clinical outcome data was not available from our electronic database, which is of fundamental importance.

The SST failure rate in GC-naïve patients without established endocrine or CNS disease was higher than might have been expected (7.5\%). The explanation for this is not clear and may relate to new diagnoses of endocrine (or other, e.g. malignancy) disease that had not been fully investigated prior to the SST being requested, although the aetiology in many remains unexplained. Importantly, some of these tests were performed in the intensive care setting where the underpinning mechanisms causing compromised adrenal reserve are still unknown.

In summary, the prevalence of adrenal insufficiency due to prescribed GCs is high and almost certainly represents the commonest cause of compromised adrenal function. Importantly, we have highlighted the potent, dose-dependent ability of inhaled GCs to suppress endogenous HPA axis function, and identified the utility of a morning cortisol level in guiding the clinician as to which patients may need dynamic assessment of adrenal reserve. Futures prospective studies are needed to accurately define the clinical consequences of adrenal suppression for patients prescribed inhaled GC therapy and determine the optimal medical management for these patients.

\section{Supplementary data}

This is linked to the online version of the paper at http://dx.doi.org/10.1530/ EJE-15-0608.

\section{Declaration of interest}

The authors declare that there is no conflict of interest that could be perceived as prejudicing the impartiality of the research reported.

\section{Funding}

This work has been supported by the Medical Research Council (Senior Clinical Fellowship ref. G0802765, J W Tomlinson), Irish Endocrine Society Travel Bursary (C P Woods) and through the Oxford NIHR Biomedical Research Centre.

\section{Author contribution statement}

C P Woods, N Argese and M Chapman analysed the data and wrote the manuscript. C Boot and R Webster collected the biochemical data and helped to write the manuscript. $\mathrm{V}$ Dabhi collected the data from the electronic patient records and analysed the data. A A Toogood, A B Grossman, W Arlt, P M Stewart and R K Crowley scrutinized, analysed and interpreted the data and helped to write the manuscript. J W Tomlinson designed the study, analysed and interpreted the data and wrote the manuscript.

\section{Acknowledgements}

We would like to thank Professor John Wass for his critical analysis of the manuscript.

\section{References}

1 Plotz CM, Knowlton AI \& Ragan C. The natural history of Cushing's syndrome. American Journal of Medicine 195213 597-614. (doi:10.1016/ 0002-9343(52)90027-2)

2 Bergthorsdottir R, Leonsson-Zachrisson M, Odén A \& Johannsson G. Premature mortality in patients with Addison's disease: a populationbased study. Journal of Clinical Endocrinology and Metabolism 200691 4849-4853. (doi:10.1210/jc.2006-0076)

3 Crowley RK, Argese N, Tomlinson JW \& Stewart PM. Central hypoadrenalism. Journal of Clinical Endocrinology and Metabolism 2014 99 4027-4036. (doi:10.1210/jc.2014-2476)

4 Arlt W \& Stewart PM. Adrenal corticosteroid biosynthesis, metabolism, and action. Endocrinology and Metabolism Clinics of North America 2005 34 293-313, viii. (doi:10.1016/j.ecl.2005.01.002)

5 Stewart PM, Corrie J, Seckl JR, Edwards CR \& Padfield PL. A rational approach for assessing the hypothalamo-pituitary-adrenal axis. Lancet 19881 1208-1210. (doi:10.1016/S0140-6736(88)92020-X)

6 Bancos I, Hahner S, Tomlinson J \& Arlt W. Diagnosis and management of adrenal insufficiency. Lancet. Diabetes \& Endocrinology 20143 216-226. (doi:10.1016/S2213-8587(14)70142-1)

7 Burman P, Mattsson AF, Johannsson G, Höybye C, Holmer H, Dahlqvist P, Berinder K, Engström BE, Ekman B, Erfurth EM et al. Deaths among adult patients with hypopituitarism: hypocortisolism during acute stress, and de novo malignant brain tumors contribute to an increased mortality. Journal of Clinical Endocrinology and Metabolism 201398 1466-1475. (doi:10.1210/jc.2012-4059) 
8 Allolio B. Extensive expertise in endocrinology. Adrenal crisis. European Journal of Endocrinology/European Federation of Endocrine Societies 2015 172 R115-R124. (doi:10.1530/EJE-14-0824)

9 Overman RA, Yeh J-Y \& Deal CL. Prevalence of oral glucocorticoid usage in the United States: a general population perspective. Arthritis Care \& Research 201365 294-298. (doi:10.1002/acr.21796)

10 Van Staa TP, Leufkens HG, Abenhaim L, Begaud B, Zhang B \& Cooper C. Use of oral corticosteroids in the United Kingdom. QJM 200093 105-111. (doi:10.1093/qjmed/93.2.105)

11 Van Staa TP, Cooper C, Leufkens HG, Lammers JW \& Suissa S. The use of inhaled corticosteroids in the United Kingdom and the Netherlands. Respiratory Medicine 200397 578-585. (doi:10.1053/rmed.2002.1453)

12 Dahl R. Systemic side effects of inhaled corticosteroids in patients with asthma. Respiratory Medicine 2006100 1307-1317. (doi:10.1016/j.rmed. 2005.11.020)

13 Agha A, Tomlinson JW, Clark PM, Holder G \& Stewart PM. The long-term predictive accuracy of the short Synacthen (corticotropin) stimulation test for assessment of the hypothalamic-pituitary-adrenal axis. Journal of Clinical Endocrinology and Metabolism 200691 43-47. (doi:10.1210/jc.2005-1131)

14 Stokes FJ, Bailey LM, Ganguli A \& Davison AS. Assessment of endogenous, oral and inhaled steroid cross-reactivity in the Roche cortisol immunoassay. Annals of Clinical Biochemistry 201451 503-506. (doi:10.1177/0004563213509793)

15 Clark PM, Neylon I, Raggatt PR, Sheppard MC \& Stewart PM. Defining the normal cortisol response to the short Synacthen test: implications for the investigation of hypothalamic-pituitary disorders. Clinical Endocrinology 199849 287-292. (doi:10.1046/j.1365-2265. 1998.00555.x)

16 Henzen C, Suter A, Lerch E, Urbinelli R, Schorno XH \& Briner VA. Suppression and recovery of adrenal response after short-term, high-dose glucocorticoid treatment. Lancet 2000355 542-545. (doi:10.1016/S0140-6736(99)06290-X)

17 Souverein PC, Berard A, Van Staa TP, Cooper C, Egberts AC, Leufkens HG \& Walker BR. Use of oral glucocorticoids and risk of cardiovascular and cerebrovascular disease in a population based casecontrol study. Heart 200490 859-865. (doi:10.1136/hrt.2003.020180)

18 Brigell DF, Fang VS \& Rosenfield RL. Recovery of responses to ovine corticotropin-releasing hormone after withdrawal of a short course of glucocorticoid. Journal of Clinical Endocrinology and Metabolism $1992 \mathbf{7 4}$ 1036-1039. (doi:10.1210/jcem.74.5.1314844)

19 Petersen KB, Müller J, Rasmussen M \& Schmiegelow K. Impaired adrenal function after glucocorticoid therapy in children with acute lymphoblastic leukemia. Medical and Pediatric Oncology 200341 110-114. (doi:10.1002/mpo.10316)

20 Schuetz P, Leuppi JD, Bingisser R, Bodmer M, Briel M, Drescher T, Duerring U, Henzen C, Leibbrandt $\mathrm{Y}$, Maier S et al. Prospective analysis of adrenal function in patients with acute exacerbations of COPD: the Reduction in the Use of Corticosteroids in Exacerbated COPD (REDUCE) trial. European Journal of Endocrinology/European Federation of Endocrine Societies 2015173 19-27. (doi:10.1530/EJE-15-0182)

21 Schlaghecke R, Kornely E, Santen RT \& Ridderskamp P. The effect of long-term glucocorticoid therapy on pituitary-adrenal responses to exogenous corticotropin-releasing hormone. New England Journal of Medicine 1992326 226-230. (doi:10.1056/NEJM199201233260403)

22 Sacre K, Dehoux M, Chauveheid MP, Chauchard M, Lidove O, Roussel R \& Papo T. Pituitary-adrenal function after prolonged glucocorticoid therapy for systemic inflammatory disorders: an observational study. Journal of Clinical Endocrinology and Metabolism 201398 3199-3205. (doi:10.1210/jc.2013-1394)

23 Mahlab-Guri K, Asher I, Gradstein S, Zung A, Radian-Sade S, Elbirt D \& Sthoeger Z. Inhaled fluticasone causes iatrogenic Cushing's syndrome in patients treated with Ritonavir. Journal of Asthma 201148 860-863. (doi:10.3109/02770903.2011.606580)

24 Hoover WC, Britton LJ, Gardner J, Jackson T \& Gutierrez H. Rapid onset of iatrogenic adrenal insufficiency in a patient with cystic fibrosis-related liver disease treated with inhaled corticosteroids and a moderate CYP3A4 inhibitor. Annals of Pharmacotherapy 201145 e38. (doi:10.1345/aph.1Q103)

25 Kedem E, Shahar E, Hassoun G \& Pollack S. Iatrogenic Cushing's syndrome due to coadministration of ritonavir and inhaled budesonide in an asthmatic human immunodeficiency virus infected patient. Journal of Asthma 201047 830-831. (doi:10.3109/02770903.2010. 485666)

26 Boyd SD, Hadigan C, McManus M, Chairez C, Nieman LK, Pau AK, Alfaro RM, Kovacs JA, Calderon MM \& Penzak SR. Influence of low-dose ritonavir with and without darunavir on the pharmacokinetics and pharmacodynamics of inhaled beclomethasone. Journal of Acquired Immune Deficiency Syndromes 201363 355-361. (doi:10.1097/QAI. 0b013e31829260d6)

27 Smith RW, Downey K, Gordon M, Hudak A, Meeder R, Barker S \& Smith WG. Prevalence of hypothalamic-pituitary-adrenal axis suppression in children treated for asthma with inhaled corticosteroid. Paediatrics \& Child Health 201217 e34-e39.

28 Allen A, Schenkenberger I, Trivedi R, Cole J, Hicks W, Gul N \& Jacques L. Inhaled fluticasone furoate/vilanterol does not affect hypothalamic-pituitary-adrenal axis function in adolescent and adult asthma: randomised, double-blind, placebo-controlled study. Clinical Respiratory Journal 20137 397-406. (doi:10.1111/crj.12026)

29 Brown PH, Blundell G, Greening AP \& Crompton GK. Hypothalamopituitary-adrenal axis suppression in asthmatics inhaling high dose corticosteroids. Respiratory Medicine 199185 501-510. (doi:10.1016/ S0954-6111(06)80268-4)

30 Holme J, Tomlinson JW, Stockley RA, Stewart PM, Barlow N \& Sullivan AL. Adrenal suppression in bronchiectasis and the impact of inhaled corticosteroids. European Respiratory Journal 200832 1047-1052. (doi:10.1183/09031936.00016908)

31 Fowler SJ, Orr LC, Wilson AM, Sims EJ \& Lipworth BJ. Dose-response for adrenal suppression with hydrofluoroalkane formulations of fluticasone propionate and beclomethasone dipropionate. British Journal of Clinical Pharmacology 200152 93-95. (doi:10.1046/j.0306-5251.2001. bjcp.1399.x)

32 Jamilloux Y, Liozon E, Pugnet G, Nadalon S, Heang Ly K, Dumonteil S, Gondran G, Fauchais AL \& Vidal E. Recovery of adrenal function after long-term glucocorticoid therapy for giant cell arteritis: a cohort study. PLOS ONE 20138 e68713. (doi:10.1371/journal.pone.0068713)

33 Suissa S, Kezouh A \& Ernst P. Inhaled corticosteroids and the risks of diabetes onset and progression. American Journal of Medicine 2010123 1001-1006. (doi:10.1016/j.amjmed.2010.06.019)

34 Cohen J \& Venkatesh B. Relative adrenal insufficiency in the intensive care population; background and critical appraisal of the evidence. Anaesthesia and Intensive Care 201038 425-436.

35 Finucane FM, Liew A, Thornton E, Rogers B, Tormey W \& Agha A. Clinical insights into the safety and utility of the insulin tolerance test (ITT) in the assessment of the hypothalamo-pituitary-adrenal axis. Clinical Endocrinology 200869 603-607. (doi:10.1111/j.1365-2265. 2008.03240.x)

36 Plumpton FS \& Besser GM. The adrenocortical response to surgery and insulin-induced hypoglycemia in corticosteroid-treated and normal subjects. British Journal of Surgery 196855 857. (doi:10.1002/bjs. 1800551113)

37 Yo WS, Toh L-M, Brown SJ, Howe WD, Henley DE \& Lim EM. How good is a morning cortisol in predicting an adequate response to intramuscular synacthen stimulation? Clinical Endocrinology 201481 19-24. (doi:10.1111/cen.12373)

38 Varadhan L, Nayak AU, Mukherjee A, Jose B \& Varughese GI. Can a baseline morning cortisol predict outcome of short Synacthen test in an endocrine unit in an outpatient setting. Clinical Endocrinology 201582 309-311. (doi:10.1111/cen.12585)

39 Hägg E, Asplund K \& Lithner F. Value of basal plasma cortisol assays in the assessment of pituitary-adrenal insufficiency. Clinical Endocrinology 198726 221-226. (doi:10.1111/j.1365-2265.1987.tb00780.x) 
40 Kazlauskaite R, Evans AT, Villabona CV, Abdu TA, Ambrosi B, Atkinson $\mathrm{AB}$, Choi $\mathrm{CH}$, Clayton RN, Courtney CH, Gonc EN et al. Corticotropin tests for hypothalamic-pituitary-adrenal insufficiency: a metaanalysis. Journal of Clinical Endocrinology and Metabolism 2008 93 4245-4253. (doi:10.1210/jc.2008-0710)

41 Le Roux CW, Meeran K \& Alaghband-Zadeh J. Is a 0900-h serum cortisol useful prior to a short synacthen test in outpatient assessment? Annals of Clinical Biochemistry 200239 148-150. (doi:10.1258/ 0004563021901919)

42 Schmidt IL, Lahner H, Mann K \& Petersenn S. Diagnosis of adrenal insufficiency: evaluation of the corticotropin-releasing hormone test and Basal serum cortisol in comparison to the insulin tolerance test in patients with hypothalamic-pituitary-adrenal disease. Journal of Clinical Endocrinology and Metabolism 200388 4193-4198. (doi:10.1210/ jc.2002-021897)

43 Kadiyala R, Kamath C, Baglioni P, Geen J \& Okosieme OE. Can a random serum cortisol reduce the need for short synacthen tests in acute medical admissions? Annals of Clinical Biochemistry 201047 378-380. (doi:10.1258/acb.2010.010008)

44 Stewart PM \& Clark P. The short Synacthen test: is less best? Clinical Endocrinology 199951 151-152. (doi:10.1046/j.1365-2265. 1999.00801.x)

Received 19 June 2015

Revised version received 6 August 2015

Accepted 20 August 2015 